\title{
LABORATORIO DE GEOCRONOLOGÍA EN EL SERVICIO GEOLÓGICO COLOMBIANO: AVANCES SOBRE DATACIÓN U-Pb EN CIRCONES MEDIANTE LA TÉCNICA LA-ICP-MS
}

\author{
GEOCHRONOLOGY LABORATORY AT THE SERVICIO GEOLÓGICO \\ COLOMBIANO (COLOMBIAN GEOLOGICAL SURVEY): ADVANCES IN THE \\ U-Pb DATING OF ZIRCONS BY THE LA-ICP-MS TECHNIOQUE
}

\author{
Mary Luz Peña Urueña ${ }^{1}$, Jimmy Alejandro Muñoz Rocha ${ }^{2}$, Cindy Lizeth Urueña ${ }^{3}$ \\ *Email de correspondencia: mlpena@sgc.gov.co
}

Recibido: 15/08/2017

Aceptado: 26/02/2018

Publicado en línea: 20/04/2018

Citación: Peña Urueña, M. L., Muñoz Rocha, J. A. y Urueña, C. L. (2018). Laboratorio de Geocronología en el Servicio Geológico Colombiano: avances sobre datación U-Pb en circones mediante la técnica LA-ICP-MS. Boletín Geológico, 44, 39-56.

\section{RES UM EN}

En este trabajo se presentan los primeros resultados de la implementación del Laboratorio de Datación U-Pb en el Servicio Geológico Colombiano con la técnica analítica LA-ICP-MS para datación $\mathrm{U}-\mathrm{Pb}$. Este método geocronológico está basado en el decaimiento radiactivo de los isótopos de Uranio $\left({ }^{238} \mathrm{U} /{ }^{206} \mathrm{~Pb}\right.$ y $\left.{ }^{235} \mathrm{U} /{ }^{207} \mathrm{~Pb}\right)$, con el fin de ser empleado en la determinación de edades en circones. Como parte del proceso, se trabajó en la evaluación de materiales de referencia con diferentes rangos de edades, utilizando parámetros analíticos similares a los que utilizan otros laboratorios de diversas partes del mundo, con el fin de obtener resultados de precisión y exactitud óptima para esta técnica analítica. Los resultados obtenidos muestran que, en general, para la mayoría de los materiales de referencia, la precisión como coeficiente de variación y veracidad, como la dife-

1 M. Sc. en Ciencias Química. Servicio Geológico Colombiano, Dirección de Asuntos Nucleares, Grupo de Investigaciones y Aplicaciones Nucleares

2 M. Sc. en Sistemas Integrados de Gestión de la Prevención de Riesgos Laborales, la Calidad, el Medio Ambiente y la Responsabilidad Social Corporativa. Servicio Geológico Colombiano, Dirección de Asuntos Nucleares, Laboratorio de Datación U/Pb

3 M. Sc. en Ciencias, Geología. Servicio Geológico Colombiano, Dirección de Asuntos Nucleares, Laboratorio Huellas de Fisión 
rencia entre la edad esperada y la obtenida, es menor del $2 \%$. Además, se evaluó la concordancia del sistema $\mathrm{U}-\mathrm{Pb}$ mediante diagramas de concordia, y se encontró que la desviación de la media estándar ponderada (MSWD), en la mayoría de los estándares, es menor de 2, lo cual implica que se obtuvieron resultados en el margen de los parámetros estadísticos establecidos.

Palabras clave: ablación, láser, espectrometría de masas, datación, geocronología, U/Pb, circones, LA-ICP-MS.

\begin{abstract}
A B S T R A C T
This paper presents the first results of the implementation of the $\mathrm{U}-\mathrm{Pb}$ dating laboratory at the Servicio Geológico Colombiano (Colombian Geological Survey) using the LA-ICP-MS analytical technique for $\mathrm{U}-\mathrm{Pb}$ dating. This geochronological method is based on the radioactive decay of the isotopes of Uranium $\left({ }^{238} \mathrm{U} /{ }^{206} \mathrm{~Pb}\right.$ and $\left.{ }^{235} \mathrm{U} /{ }^{207} \mathrm{~Pb}\right)$ in order to be used in the zircon age determinations. As part of the implementation process, we evaluated reference materials with different age ranges using analytical parameters similar to those used by other laboratories worldwide with the aim of obtaining results with optimal precision and accuracy for this analytical technique. In general, the results obtained show that for the majority of the reference materials, the precision as the coefficient of variation and the veracity as the difference between the expected age and the obtained age are less than $2 \%$. In addition, we evaluated the concordance of the U-Pb system using concordance diagrams and we found that the mean square weighted deviation (MSWD) in most standards were less than 2, implying that the results were obtained within the established statistical parameters.
\end{abstract}

Keywords: Ablation, laser, mass spectrometry, dating, geochronology, U/Pb, zircon, LA ICP-MS.

\section{INTRODUCCIÓN}

ntre los métodos de datación para materiales geológicos, el sistema isotópico U-Th-Pb es uno de los más importantes y utilizados para determinar edades absolutas, debido a la abundancia de minerales con altos contenidos de $\mathrm{U}$ en la mayoría de los tipos de rocas (p. ej., sedimentarias, metamórficas, ígneas plutónicas alcalínas y volcánicas) y a la resistencia fisicoquímica de dichos minerales (p. ej., circón [ $\mathrm{ZrSiO} 4])$. Además, permite la datación de eventos geológicos (p. ej., emplazamiento de intrusivos, vulcanismo, metamorfismo, entre otros eventos tectonotermales) ocurridos desde hace 4,57 Ga hasta el Pleistoceno (Schoene, 2014). El circón es el mineral más ampliamente utilizado, porque en él se registran eventos de alta temperatura (p. ej., cristalización o metamorfismo de alto grado), ya que tiene una temperatura de cierre para este sistema isotópico mayor de $800{ }^{\circ} \mathrm{C}$ (Cherniak y Watson, 2001; Moser y Scott, 2001; Mattinson, 2005).

La determinación de edades se basa en la medición de isótopos que decaen radiactivamente, como es el caso del ${ }^{238} \mathrm{U}$ (isótopo padre), que decae a un isótopo radiogénico como el ${ }^{206} \mathrm{~Pb}$. La determinación de esta relación isotópica permite obtener la edad de temperatura de cierre en dicho sistema isotópico en un mineral específico (Schoene, 2014). Además, es ampliamente usada en aquellos minerales cuya concentración y relaciones isotópicas son adecuadas para determinaciones geocronológicas (Putirka, 2015), pues proporciona una variedad de aplicaciones orientadas a cartografía geológica, petrogénesis, evolución de sistemas orogénicos, exploración de recursos minerales y de recursos energéticos. Además, permite datar eventos de cristalización de rocas, eventos térmicos de alta temperatura, determinaciones de máxima edad de depositación en una cuenca sedimentaria y análisis de proveniencia, entre otros (Kosler y Sylvester, 2003; Kosler, 2007; Putirka, 2015).

En los últimos años se ha incrementado el número de publicaciones y laboratorios que a escala mundial utilizan la técnica de datación U-Th-Pb mediante ablación láser unido a espectrometría de masas de acoplamiento inductivo a plasma (LA-ICP-MS, por sus siglas en inglés), ya sea con instrumentos mono o multicolector (Paton, Woodhead, Hellstrom, Hergt, Greig y Maas, 2010; Schoene, 2014; Horstwood, Kosler, Gehrels, Jackson y McLean, 2016). Esto se debe a que estos análisis in situ en los que 
se realizan ablaciones a escalas de $\mu \mathrm{m}$ sobre la superficie de cristales pulidos, brindan resultados rápidos y precisos (Campbell, Reiners y Allen, 2005; Solari, Gómez-Tuena, Bernal, Pérez-Arvizu y Tanner, 2010).

Teniendo en cuenta que diferentes autores han reportado rutinas de laboratorios con la técnica de LA-ICP-MS (e.g. Jackson, Pearson, Griffin y Belousova, 2004; Chang Vervoort, McClelland y Knaack, 2006; Gehrels, Valencia y Ruiz, 2008; Solari et al., 2010; Bouman et al., 2014) y han especificado los parámetros analíticos que usan con el equipo de ablación láser y con el espectrómetro de masas, ya sea de tipo sector magnético o cuadrupolar, se presentan en este trabajo los resultados de ensayos preliminares obtenidos durante la fase inicial de implementación del Laboratorio de Datación U-Pb utilizando la técnica de LA-ICP-MS en el Servicio Geológico Colombiano. Esta labor se adelantó utilizando materiales de referencia que han sido previamente analizados por métodos de dilución isotópica y espectrometría de masas de ionización termal (ID-TIMS), y que posteriormente han sido evaluados en varios laboratorios a escala mundial que utilizan la LA-ICP-MS. Los materiales de referencia fueron analizados con los parámetros analíticos escogidos, y se evaluaron los resultados analíticos de cada material de referencia mediante la veracidad (comparación de resultados contra un valor de referencia), expresada como porcentaje de error, y la precisión (variabilidad de una serie de medidas), expresada como coeficiente de variación para el sistema ${ }^{206} \mathrm{~Pb} /{ }^{238} \mathrm{U}$. Finalmente, los diagramas de concordia son utilizados como herramienta gráfica para comparar las relaciones isotópicas entre los sistemas ${ }^{206} \mathrm{~Pb} /{ }^{238} \mathrm{U}$ y ${ }^{207} \mathrm{~Pb} /{ }^{235} \mathrm{U}$, con lo cual se obtiene la edad de concordia entre los dos sistemas isotópicos (Wetherill, 1956) y el valor MSWD (Wendt y Carl, 1991), que permite definir el promedio ponderado de los datos y si su incertidumbre representa una sola población (Spencer, Kirkland y Taylor, 2015).

\subsection{Implementación}

En el año 2009 el Servicio Geológico Colombiano dio inicio a la consolidación del Centro de Dataciones mediante el convenio SGC-Colciencias-CIF, financiado por la Agencia Nacional de Hidrocarburos. En una primera fase se realizó la implementación del Laboratorio de Termocronología con la técnica de huellas de fisión, aprovechando la existencia del reactor nuclear. Posteriormente se inició la implementación del Laboratorio de Datación $\mathrm{U}-\mathrm{Pb}$ con tecnología de última generación, y se adquirieron los equipos de ablación láser y espectrómetro de masas de acoplamiento inductivo a plasma.

\section{Descripción de LA METOdología}

\subsection{Principio del método}

La datación radiométrica $\mathrm{U}-\mathrm{Pb}$ se basa en el sistema U-Th-Pb. El U y el Th, encontrados de forma natural en algunos minerales, decaen de ${ }^{238} \mathrm{U}$ al isótopo estable ${ }^{206} \mathrm{~Pb}$, de ${ }^{235} \mathrm{U}$ a ${ }^{207} \mathrm{~Pb}$ y el ${ }^{232} \mathrm{Th}$ a ${ }^{208} \mathrm{~Pb}$. El tiempo de vida media para el ${ }^{238} \mathrm{U},{ }^{235} \mathrm{U}$ y ${ }^{232} \mathrm{Th}$ corresponde a $4,468 \times 10^{9}$ años, $0,7038 \times 10^{9}$ años y $1,401 \times 10^{10}$ años, respectivamente (Schoene, 2014). El cálculo de la edad se hace mediante la ecuación de actividad que relaciona la concentración de los isótopos del sistema de decaimiento de interés (Sylvester, 2003; Lee, 2012):

$$
t=\frac{1}{\lambda} \ln \left[\delta_{i m}-\frac{\left(N_{d}-N_{d_{0}}\right)}{N_{p}}+1\right]
$$

$N_{d}$ : cantidad de isótopos hijos en un tiempo t

$N_{d 0}$ : cantidad inicial de isótopos hijos

$N_{p}$ : cantidad del isótopo padre

$\lambda$ : constante de decaimiento

En un sistema ideal, usando cualquiera de los tres geocronómetros mencionados arriba con su respectiva constante de decaimiento, la edad obtenida debería ser la misma (i. e., un sistema "concordante"). Sin embargo, cuando esto no ocurre, puede deberse a a) pérdidas de $\mathrm{Pb}$ en la relación isotópica, es decir que, tras el decaimiento, los átomos de $\mathrm{Pb}$ radiogénico no corresponden a la cantidad de átomos de $\mathrm{U}$ decaídos debido a pérdida por remobilización, posterior a la cristalización o cierre de este sistema isotópico (Kosler y Sylvester, 2003; Kosler, 2007); b) presencia de $\mathrm{Pb}$ que no es producto de la desintegración del $\mathrm{U}$; este tipo de $\mathrm{Pb}$ se denomina $\mathrm{Pb}$ común $\left(\mathrm{o} \mathrm{Pb}_{\mathrm{C}}\right)$, y generalmente se evalúa midiendo la cantidad $\mathrm{de}^{204} \mathrm{~Pb}$ presente en el mineral, ya que este isótopo particular no es radiactivo ni tiene un componente radiogénico. En casos en que una cantidad de ${ }^{204} \mathrm{~Pb}$ puede ser medida, es posible implementar correcciones para estimar la contribución en las relaciones isotópicas del $\mathrm{Pb}$ no radiogénico (Paton et al., 2010; Schoene, 2014). 


\subsection{Equipos}

El Laboratorio de Geocronología U-Th-Pb del Servicio Geológico Colombiano utiliza un equipo de ablación láser unido a espectrometría de masas de acoplamiento inductivo a plasma para realizar las dataciones. A continuación se hace una breve descripción de los equipos:

\section{Sistema de ablación láser}

Láser tipo excímero (dímero excitado) de pulso corto y con longitud de onda de $193 \mathrm{~nm}$ (ultravioleta), generado por un sistema ATLEX-I-LR ${ }^{\circledR}$, refrigerado por aire. Se encuentra instalado en un sistema de trasmisión Photon Machines ${ }^{\oplus}$, modelo Excite (figura 1), con unidad de purga de nitrógeno. El láser se genera utilizando una mezcla de fluoruro de argón (ArF).

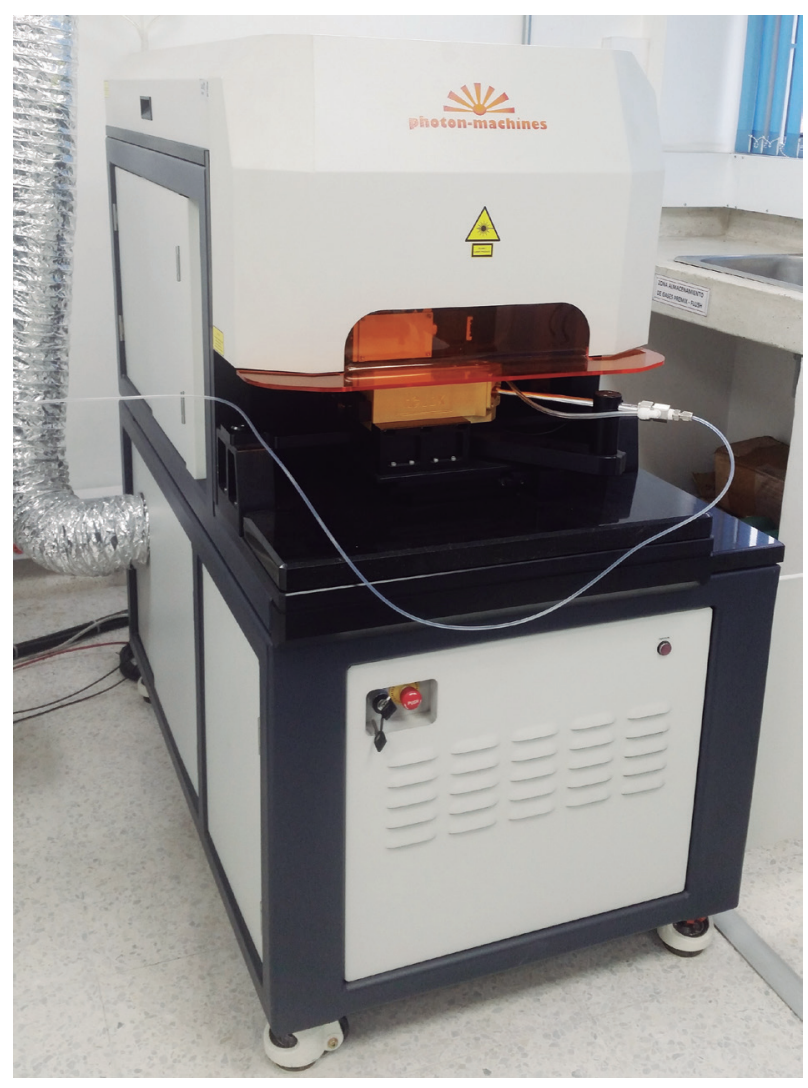

Figura 1. Equipo de ablación láser Photon Machines • modelo Excite de $193 \mathrm{~nm}$

Fuente: Laboratorio de Geocronología, Servicio Geológico Colombiano
Los montajes con los circones que se quieren datar se colocan en un portamuestras (figura 2, abajo) el cual se introduce dentro de una celda de doble volumen HelEx, purgada y presurizada con helio. El gas de arrastre que va hacia el espectrómetro se ajusta utilizando controladores de flujo de masa de marca Alicat ${ }^{\bullet}$ (MFC4 y MFC2). El primero presuriza la celda Helex y arrastra el aerosol generado durante la ablación; el segundo presuriza la cámara de ablación y arrastra el aerosol generado hacia el espectrómetro de masas. El portamuestras cuenta con espacio para probetas y secciones delgadas de diferente tamaño (figura 2, abajo).
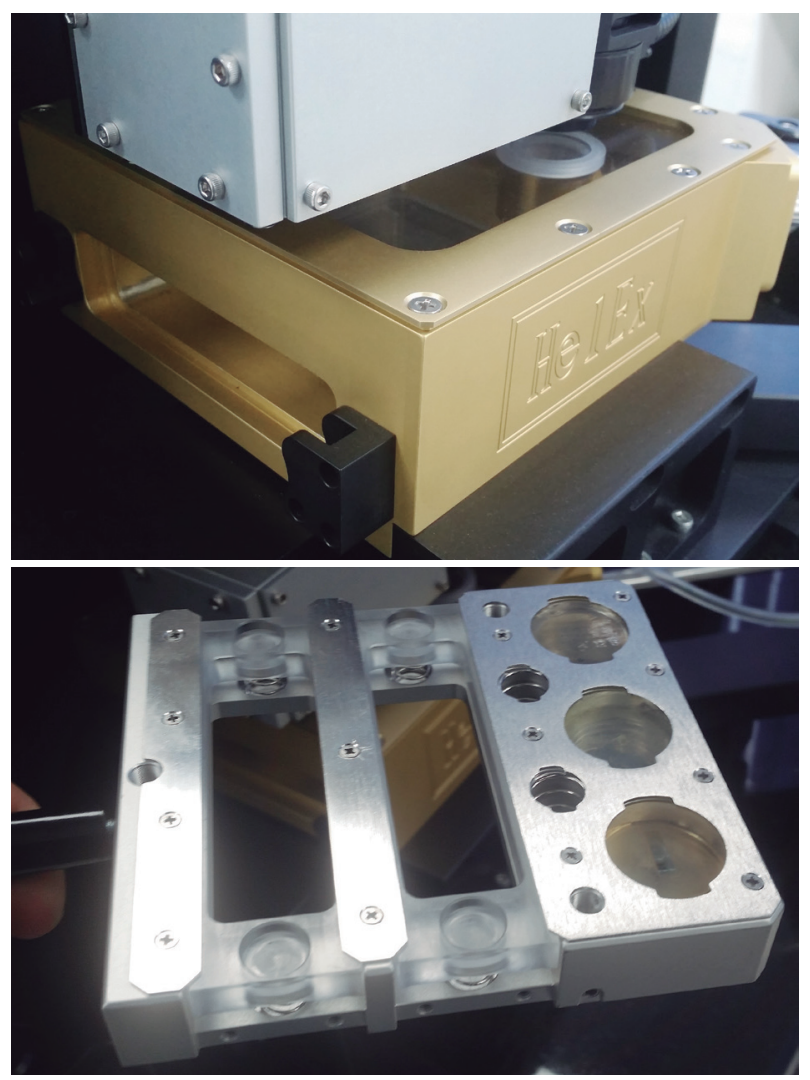

Figura 2. Arriba, celda de doble volumen y videocámara. Abajo, portamuestras

Fuente: Laboratorio de Geocronología, Servicio Geológico Colombiano

La vista de muestra durante la ablación es proporcionada por una videocámara situada fuera del eje y conectada a un monitor externo (figura 2, izquierda). El sistema de ablación con láser se acopló a un ICP-MS de sector magnético por medio de un homogeneizador de gases tipo "pulpo" de ocho brazos (figura 3) con el fin de suavizar las señales. 


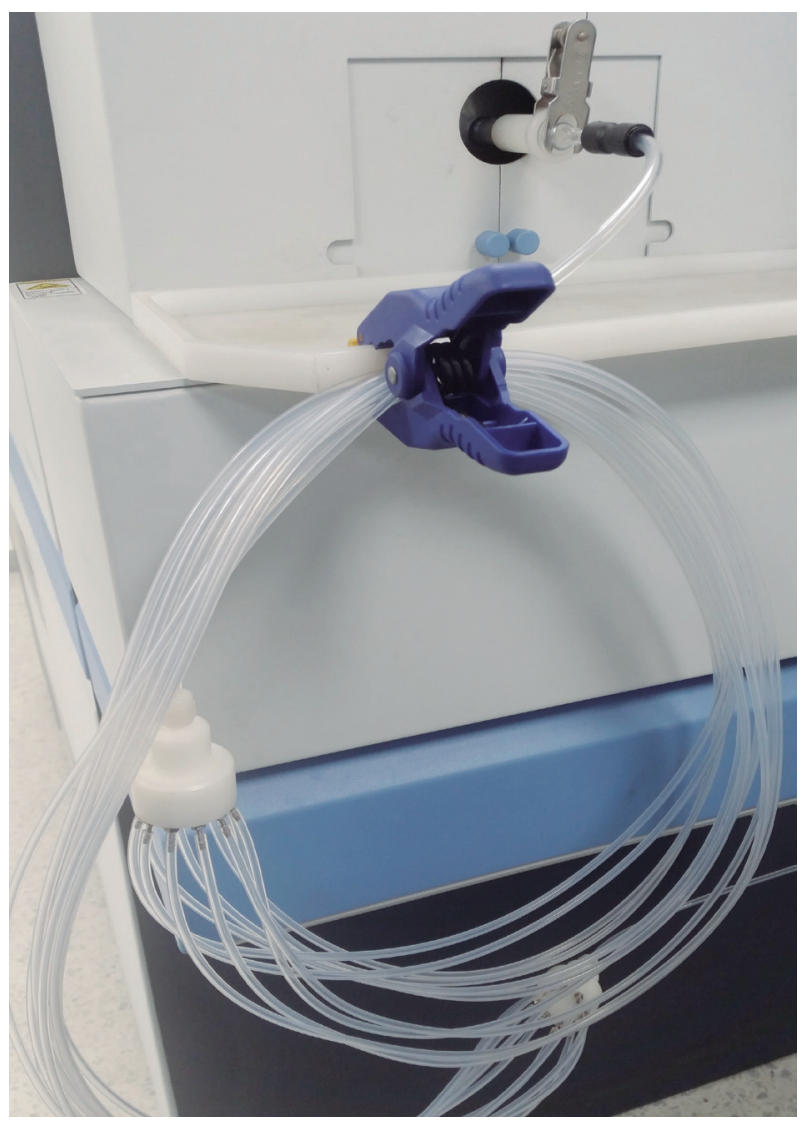

Figura 3. Sistema de ocho brazos (squid o pulpo), homogeneizador del aerosol de la muestra arrastrada con He hacia el ICP-MS Fuente: Laboratorio de Geocronología, Servicio Geológico Colombiano

\section{Espectrómetro de masas de acoplamiento inductivo}

\section{a plasma}

Consiste en un equipo de sector magnético, modelo Element 2 (figura 3), de la marca Thermo Scientific ${ }^{\circ}$. En él se realiza la separación de iones como función de su masa usando un magneto y un separador electrostático. Este cuenta con un único detector (multiplicador de electrones secundarios de dinodos discretos) que capta señales iónicas en el rango de $10^{1}$ a $10^{9}$ cuentas o impactos iónicos por segundo (cps). Las señales de $10^{1}$ hasta $\sim 10^{6} \mathrm{cps}$ son procesadas utilizando los 16 dinodos del multiplicador y un contador de pulsos (modo "conteo de pulsos"), mientras que las de $10^{6}$ hasta $10^{9}$ cps son medidas usando un capacitor (modo "análogo"). El modo análogo es calibrado con respecto a la señal de "conteo", y usa un factor de corrección análoga (o ACF) medido en señales en el rango de $\sim 10^{5}$ a $10^{6} \mathrm{cps}$.

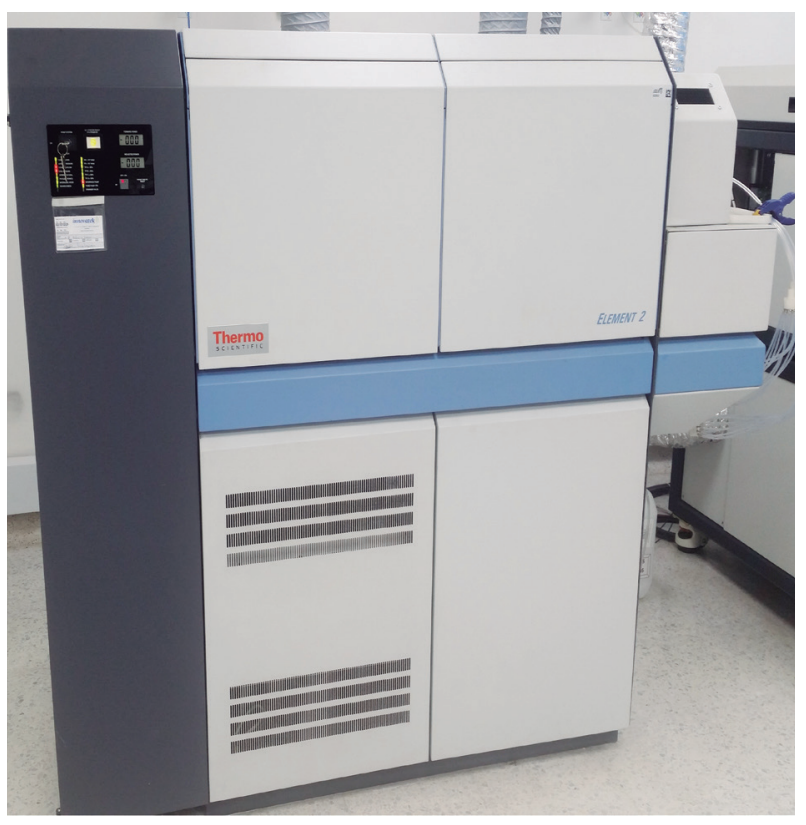

Figura 4. Espectrómetro de masas de acoplamiento inductivo a plasma. Modelo Element 2

Fuente: Laboratorio de Geocronología, Servicio Geológico Colombiano

El laboratorio cuenta con condiciones ambientales controladas para mantener la temperatura entre $18{ }^{\circ} \mathrm{C} \mathrm{y}$ $24^{\circ} \mathrm{C}$, y una humedad relativa entre $50 \%$ HR y $60 \% \mathrm{HR}$. Además, un recirculador de agua Thermo Flex ${ }^{\circ}$ que refrigera el sector magnético del Element 2.

\subsection{Insumos}

\section{Gases}

Gases $\mathrm{He}, \mathrm{N}_{2}$ y Ar de ultraalta pureza y mezcla gaseosa de ArF Gas premix (para el láser Excímer de 193 nm).

\section{Materiales de referencia}

Solución de sintonización Thermo Scientific ${ }^{\bullet}$ de $1 \mu \mathrm{g} / \mathrm{L}$ y Vidrio NIST 612 (Pearce, Perkins, Westgate, Gorton, Jackson y Neal, 1997).

Los materiales de referencia utilizados para los análisis se describen a continuación: 
Tabla 1. Materiales de referencia utilizados para los análisis

\begin{tabular}{cc}
\hline Estándar/referencia & Edad (Ma.)* \\
\hline $\begin{array}{c}\text { Plešovice } \\
\text { (Sláma Kosler, Condon, Crowley, Gerdes y Hanchar, 2008) }\end{array}$ & $337,13 \pm 0,37$ \\
\hline Fisch Canyon Tuff (Lanphere y Baadsgaard, 1999) & $28,402 \pm 0,023$ \\
\hline FC1 (Paces y Miller, 1993) & $1099 \pm 0,6$ \\
\hline Mount Dromedary (Renne, Swisher, Deino, Karner, Owens y \\
DePaolo, 1998) & $99,12 \pm 0,14$ \\
\hline Mud Tank (Black y Gulson, 1978) & $732 \pm 5$ \\
\hline R33 (Black, Kamo y Allen,2004) & $419,26 \pm 0,39$ \\
\hline WF-1 $*$ & 590 \\
\hline WF-10 $\%$ & 530 \\
\hline WF- 2 $*$ & 590 \\
\hline WF- 6 $\%$ & 530 \\
\hline
\end{tabular}

* Las edades en millones de años (Ma.) reportadas son las determinadas mediante las técnicas utilizadas en los respectivos artículos de referencias.

** Circones de Wildfish Gems, Sri Lanka. Materiales suministrados por la Universidad de California, en Santa Cruz (UCSC),

EE. UU., utilizados como materiales de referencia internos del laboratorio.

Fuente: autores

\subsection{Parámetros analíticos}

En la implementación se adoptaron algunos parámetros (frecuencia de la ablación, fluencia o energía y tamaño de ablación, descritos en la tabla 2) similares a los utilizados en otros laboratorios cuando se emplea la técnica LA-ICP-MS, tal como se explicó.

Tabla 2. Parámetros utilizados en el equipo de ablación láser

\begin{tabular}{cc}
\hline Parámetro & Valor \\
\hline Tiempo de análisis por punto de ablación* & $1,05 \mathrm{~min}$ \\
\hline Tiempo de ablación & $32 \mathrm{~s}$ \\
\hline Flujo de gas He MFC 1 & $0,540 \mathrm{~L} / \mathrm{min}$ \\
\hline Flujo de gas He MFC 2 & $0,540 \mathrm{~L} / \mathrm{min}$ \\
\hline Fluencia & $7,00 \mathrm{~J} / \mathrm{cm}{ }^{2}$ \\
\hline Láser output*** & $50 \%$ \\
\hline Frecuencia & $8 \mathrm{~Hz}$ \\
\hline Tamaño de ablación & $30 \mu \mathrm{m}$ \\
\hline
\end{tabular}

* Tiempo de análisis por punto de ablación: este tiempo incluye el tiempo que dura la ablación más la adquisición del blanco.

* El láser output es el porcentaje de atenuación de la energía del láser, para nuestro caso $50 \%$. Vale la pena aclarar que estos parámetros están en constante proceso de optimización, lo cual implica que los valores usados para generar los resultados de este reporte no son necesariamente los mismos que se utilizan actualmente.

Fuente: autores

\subsection{Rutina analítica}

Se realizó un montaje (probeta) con resina epóxica en el que se colocaron aproximadamente entre quince a veinte circones de cada material de referencia. Luego, el montaje fue pulido con diferentes lijas hasta llegar a la fase final, en la que se pulió con pasta de diamante de $6 \mu \mathrm{m}$. El montaje fue lavado en ultrasonido con agua desionizada y limpiado nuevamente con metanol grado reactivo analítico para remover grasas y aceites de la superficie (Paton et al., 2010).

Las etapas que se realizan en la rutina analítica se describen a continuación:

\section{Sintonización ICP-MS}

La sintonización del sistema LA-ICPMS se realiza, en primera instancia, usando un sistema nebulizador de líquidos, con lo cual se logra aspirar la solución de sintonización de concentración de $1 \mu \mathrm{g} / \mathrm{L}$ para los elementos Li, $\mathrm{La}, \mathrm{In}, \mathrm{U}$, entre otros, con el fin de realizar la calibración de masas y verificación de estabilidad de la señal, en la que se encuentra una media de dispersión como desviación estándar relativa para mediciones de ${ }^{139} \mathrm{La},{ }^{115} \mathrm{In} \mathrm{y}{ }^{238} \mathrm{U}<\mathrm{a} 1$, intensidades para $U$ de aproximadamente $2 \times 10^{6} \mathrm{cps}$, In $2 \times 10^{6}$ cps y La 5 c $10^{5}$ cps.

Luego, se retira el nebulizador de líquidos y se coloca el sistema de transporte de gases del láser, incluido el homogeneizador (pulpo), como se observa en la figura 3, y se sintonizan el espectrómetro y el láser ablacionado sobre el vidrio NIST 612 (Pearce et al., 1997), a lo largo de una línea (raster) utilizando un tamaño de spot de $40 \mu \mathrm{m}$, con una taza de repetición de $10 \mathrm{~Hz}$ y una fluencia de $7,0 \mathrm{~J} / \mathrm{cm}^{2}$. La sintonización se logra ajustando parámetros de la antorcha en las posiciones X, Y y Z, flujo de gas de muestra, flujo de gas auxiliar, flujo gas He de arrastre, entre otros, hasta lograr intensidades para el ${ }^{238} \mathrm{U}$ mayores de 400000 cps, y para el ${ }^{232} \mathrm{Th}$, mayores de $250000 \mathrm{cps}$. También se monitorean los óxidos de Torio de 264 unidades de masa atómica $\left(\mathrm{ThO}_{2}\right)$, manteniendo una relación $\mathrm{ThO}_{2} / \mathrm{Th}$ no superior a $0,1 \%$. Además, se monitorea la intensidad de los sisótopos de ${ }^{202} \mathrm{Hg}<$ a 1000 cps y ${ }^{204}(\mathrm{Hg}+\mathrm{Pb})<600$ cps (Solari et al., 2010).

\section{Creación secuencias y análisis}

Para realizar la rutina analítica, el laboratorio emplea tres materiales de referencia, y, según el orden en que se po- 
sicionan en la secuencia, reciben los nombres de patrón primario, secundario y terciario. El patrón primario es empleado para normalizar las señales y corregir las relaciones isotópicas de las muestras por fraccionamiento de excavación (i. e., down-pit fractionation) en función del modelo de fraccionamiento isotópico que presente el patrón primario (Paton et al., 2010; Schoene, 2014). Los llamados patrones secundarios y terciarios se utilizan para controlar las correcciones que se realizan a partir del patrón primario. Las edades promedio obtenidas para los patrones secundarios y terciarios se evalúan como muestras desconocidas, y se verifica su exactitud y precisión con respecto a la edad conocida (edad esperada) de estos materiales de referencia (Horstwood, 2008; Klotzli, Klotzli, Gunes y Kosler, 2009).

Para crear la secuencia de análisis se utiliza la técnica de bracketing, patrón-muestra-patrón, en la que se utiliza el Plešovice como patrón primario, y FC-5 Duluth, Fish Canyon Tuff o Mount Dromedary como patrones secundarios y terciarios, respectivamente, según su ubicación en la secuencia (Paton et al., 2010). Luego se magnifica la muestra que se quiere analizar con un zoom de $50 \mathrm{X}$, se realiza el correspondiente mosaico para la selección de puntos de análisis sobre los circones y se crea la secuencia de análisis en el software Chromiun 2.1 del equipo láser.

El análisis comienza haciendo incidir el láser sobre el circón para generar un aerosol que contiene los elementos e isótopos de interés. El aerosol generado es arrastrado hacia el ICP-MS mediante He ultralimpio que pasa a través del sistema homogeneizador (figura 3), y luego al espectrómetro de masas. El aerosol generado durante la ablación interactúa con el plasma (generado a partir de Ar y una alta radiofrecuencia) para formar iones que son, luego, acelerados a $6 \mathrm{kV}$ hacia el sector magnético, para separarlos en función de su diferente masa y carga usando el separador magnético y electrostático. Al final del tubo de vuelo, los iones son detectados en el multiplicador electrónico (SEM), que trasforma la señal iónica en intensidad (cuentas por segundo [cps]) para cada isótopo medido.

\section{Reducción de datos}

La señal obtenida de cada isótopo es integrada y reducida mediante el programa Iolite v2.5 ${ }^{\circ}$ en IGORPro6.3.6.4 ${ }^{\circ}$ (Paton et al., 2010; Hellstrom, 2008). Allí se efectúan las integraciones de área. Para esto, a las señales obtenidas de los isótopos de interés $\left({ }^{202} \mathrm{Hg},{ }^{204} \mathrm{Hg},{ }^{204} \mathrm{~Pb},{ }^{206} \mathrm{~Pb},{ }^{207} \mathrm{~Pb}\right.$, ${ }^{208} \mathrm{~Pb},{ }^{232} \mathrm{Th} \mathrm{y}{ }^{238} \mathrm{U}$ ) se les resta el intervalo correspondiente a la línea base. Luego se determinan las relaciones isotópicas para establecer la edad. El fraccionamiento isotópico down-pit en las relaciones ${ }^{206} \mathrm{~Pb} /{ }^{238} \mathrm{U},{ }^{207} \mathrm{~Pb} /{ }^{235} \mathrm{U}$ y ${ }^{208} \mathrm{~Pb} /{ }^{232} \mathrm{Th}$ es corregido con el patrón primario Plešovice utilizando una ecuación de tipo exponencial. El ${ }^{235} \mathrm{U}$ se calcula a partir del ${ }^{238} \mathrm{U}$ usando la relación ${ }^{238} \mathrm{U} /{ }^{235} \mathrm{U}$ igual a 137.818 (Jaffey, 1971). Al finalizar esta etapa se exportan los datos junto con los errores de propagación e internos al programa de visualización de datos Isoplot V3.75 ${ }^{\circ}$ (Ludwig, 2012) para elaborar los diagramas de concordia y las distribuciones de edad mediante histogramas.

\subsection{Metodología de implementación}

Los parámetros analíticos bajo los cuales se operaron el espectrómetro de masas y el sistema de ablación láser en este estudio son parámetros iniciales con los que se evaluaron materiales de referencia, lo cual sirvió para avanzar en el desarrollo de la técnica analítica (tabla 2).

En primera instancia, se evaluó la exactitud de la técnica en función de la edad esperada para el sistema ${ }^{206} \mathrm{~Pb} /{ }^{238} \mathrm{U}$ en cada material de referencia como función del porcentaje de error en la edad medida (Eq. 1). Esto indica qué tan lejano o cercano está el promedio de edad obtenida de cada material de referencia respecto a la edad esperada (Euraquem, 2016):

$\%$ error $=(($ edad obtenida-edad esperada $) /$ edad esperada $) * 100 \%$

Para evaluar la precisión y reproducibilidad de la técnica se analizaron aproximadamente quince ablaciones sobre los cristales de cada material de referencia, y en cada ablación se calculó la edad aparente de ${ }^{206} \mathrm{~Pb} /{ }^{238} \mathrm{U}$. Posteriormente se calculó el porcentaje de coeficiente de variación relativa (Eq. 2, relative standard deviation), que indica la dispersión de las edades obtenidas de cada material de referencia respecto a un promedio (Euraquem, 2016):

$\% \mathrm{CV}=(($ desviación estándar $) /$ promedio $) * 100 \%$

Los materiales de referencia Fish Canyon Tuff, Mud Tank, R33, WF-1, WF-2, WF-6 y WF-10 fueron evaluados 
como muestras desconocidas siguiendo el procedimiento descrito en el apartado "Creación de secuencias". En cada material de referencia evaluado como muestra se realizaron un total de 15 ablaciones, para lo cual se utilizaron en total 32 ablaciones de patrón primario Plešovice, 24 de patrón secundario FC-5 y 24 de patrón terciario Mount Dromedary. Los resultados fueron procesados de acuerdo con la metodología descrita en el apartado "Reducción de datos".

Además, los resultados se representan en los diagramas de concordia introducidos por Wetherill (1956). Para revisar las edades obtenidas por los sistemas ${ }^{206} \mathrm{~Pb} /{ }^{238} \mathrm{U}$ y ${ }^{207} \mathrm{~Pb} /{ }^{235} \mathrm{U}$ se construyeron los diagramas de concordia, en los que la edad de concordia indica la edad obtenida por promedio de los dos sistemas. La reproducibilidad de cada material de referencia es evaluada con la media cuadrática de desviaciones ponderadas (MSWD), también conocida como estadístico de Chi cuadrado reducido (Wendt y Carl, 1991; Spencer et al., 2015), que indica cuán reproducibles son las relaciones isotópicas obtenidas en cada material, considerando las incertidumbres asignadas a cada medición (Klotzli et al., 2009; Ludwig, 2012; Spencer et al., 2015). Un valor de MSWD = 1 implica que la dispersión de las medias obtenidas está perfectamente explicada por la incertidumbre analítica (asumien-

\section{a. FC 5 Duluth (patrón secundario)}

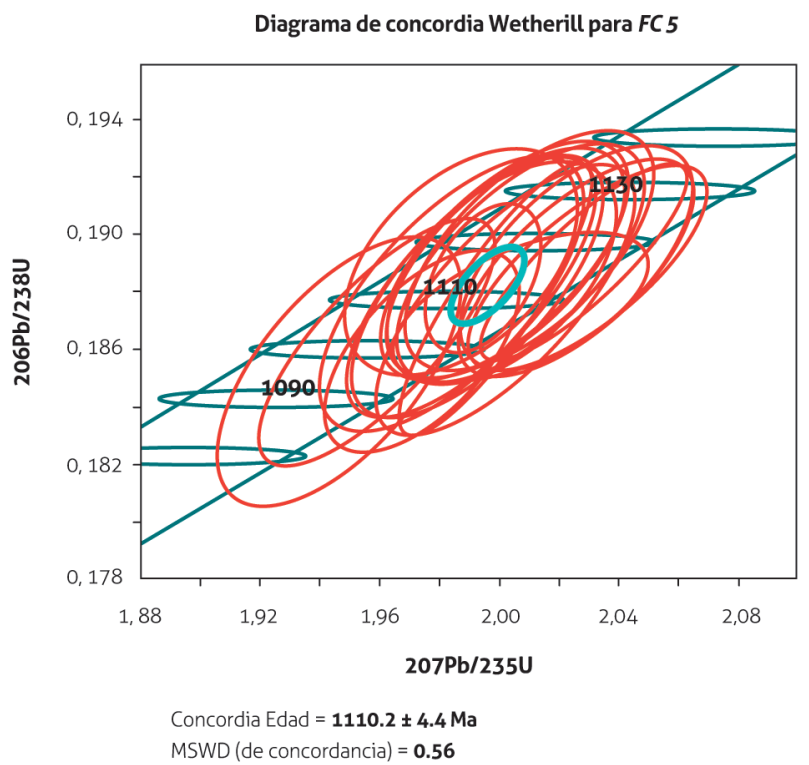

do una distribución gaussiana). El valor de $M S W D$ es $>1$ sugiere que el valor medido es mayor que el esperado, lo que indica que las incertidumbres están siendo subestimadas. Un MSWD $<1$ revela que la dispersión de las medias es mucho menor que aquella esperada, dadas las incertidumbres analíticas asignadas, esto advertiría que el error experimental probablemente está siendo sobrestimado (Klotzli et al., 2009; Ludwig, 2012; Spencer et al., 2015). Sin embargo, un MSWD $<2$ es aceptado a nivel mundial y es considerado correcto.

\section{ResUltAdOS Y ANÁLISIS}

Los resultados de los materiales de referencia se presentan a continuación en las figuras 5 a 13, donde se muestra el comportamiento estadístico de los datos. El histograma representa, de forma generalizada, el conjunto de datos e informa si su distribución es normal y si representan una misma población de circones. Finalmente, el diagrama de concordia brinda información sobre la dispersión de los datos y la exactitud de los mismos respecto a los dos sistemas independientes ${ }^{238} \mathrm{U} /{ }^{206} \mathrm{~Pb}$ y ${ }^{235} \mathrm{U} /{ }^{207} \mathrm{~Pb}$ (Wetherill, 1956; Spencer et al., 2015). En la tabla 3 se resumen los resultados estadísticos con errores internos y propagados obtenidos de los materiales de referencia evaluados.

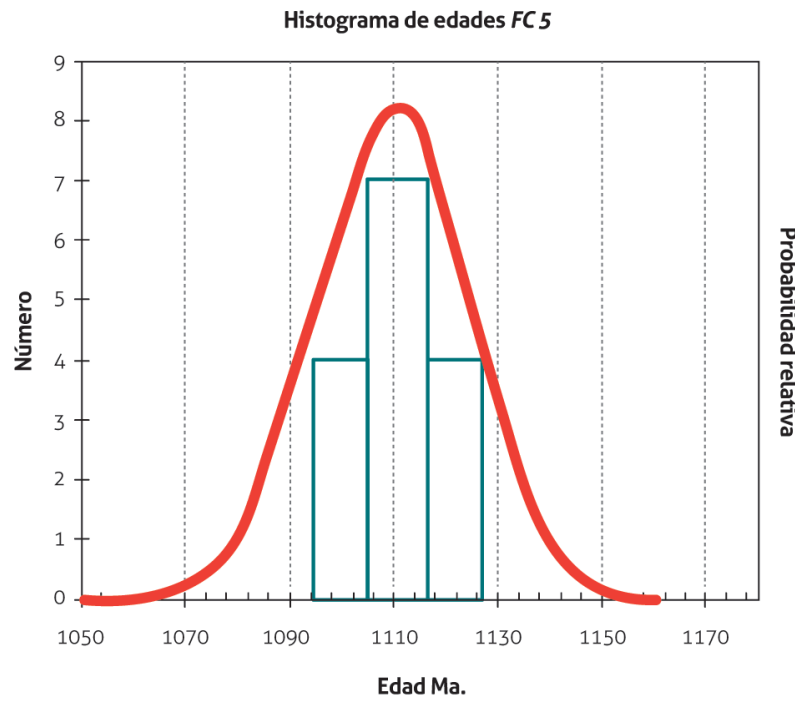

Figura 5. Izquierda, diagrama de concordia Wetherill. Derecha, histograma de edades en material de referencia FC 5 Duluth, según el sistema ${ }^{206} \mathrm{~Pb} /{ }^{238} \mathrm{U}$

Fuente: autores 


\section{b. Mount Dromedary (patrón terciario)}
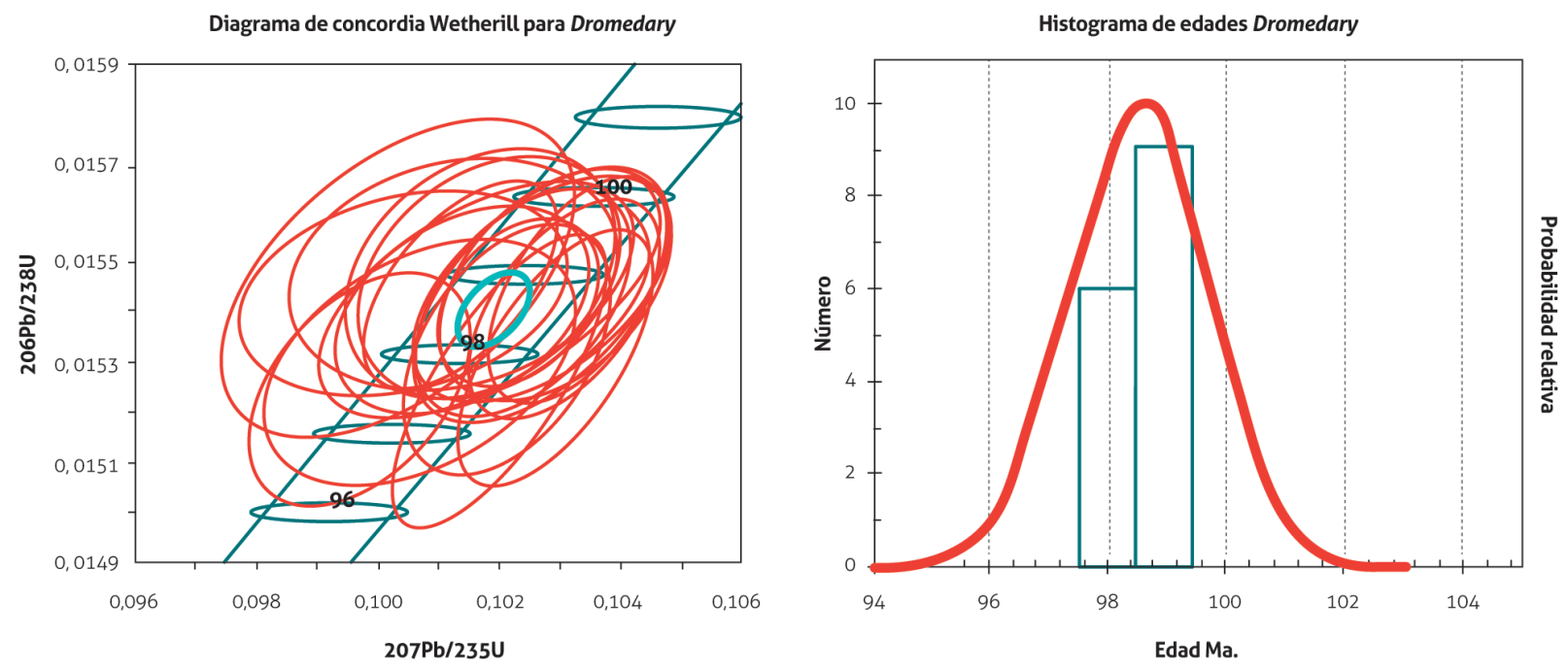

Concordia Edad $=\mathbf{9 8 . 5 3} \pm 0.33 \mathrm{Ma}$

MSWD (de concordancia) $\mathbf{0} \mathbf{0 . 0 0 2 3}$

Figura 6. Izquierda, diagrama de concordia Wetherill. Derecha, histograma de edades en material de referencia Mount Dromedary, según el sistema ${ }^{206} \mathrm{~Pb} /{ }^{238} \mathrm{U}$

Fuente: autores

\section{c. Mud Tank}
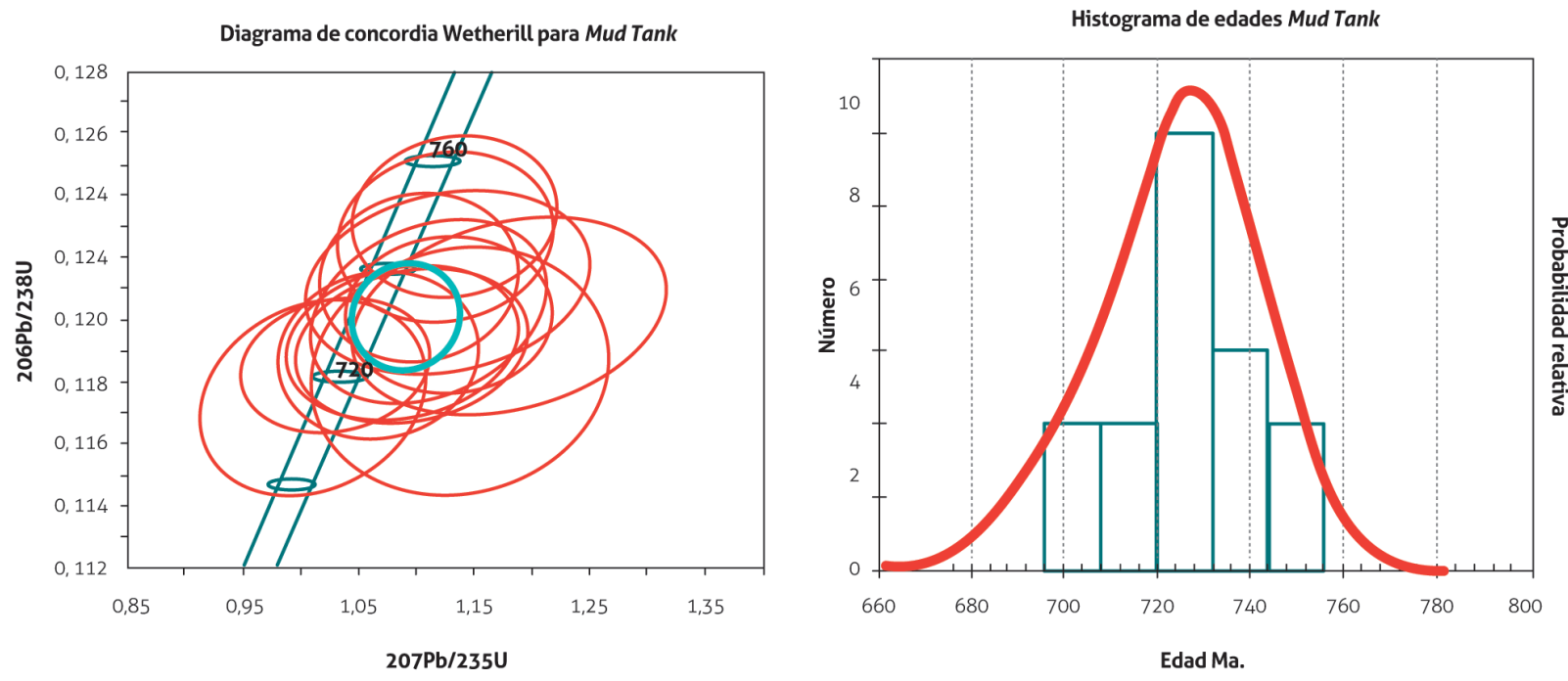

Concordia Edad $=732-2 \pm 5.9 \mathrm{Ma}$

MSWD (de concordia) $=6.4$

Figura 7. Izquierda, diagrama de concordia Wetherill. Derecha, histograma de edades en material de referencia Mud Tank, según el sistema ${ }^{206} \mathrm{~Pb} /{ }^{238} \mathrm{U}$

Fuente: autores 
d. $R 33$

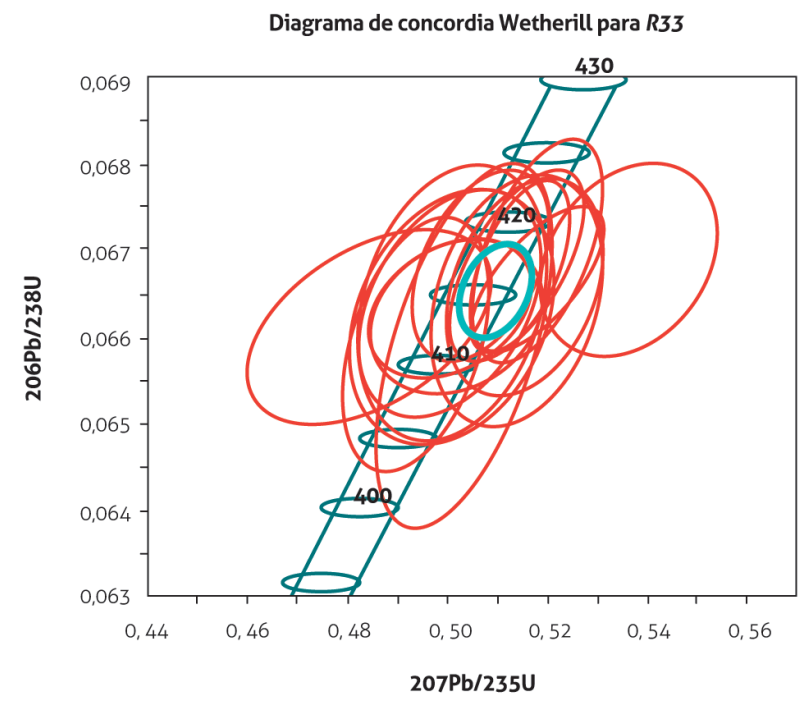

Concordia Edad $=\mathbf{4 1 5 . 4 \pm 2 . 1 \mathrm { Ma }}$

MSWD (de concordia) $=\mathbf{1 . 3}$

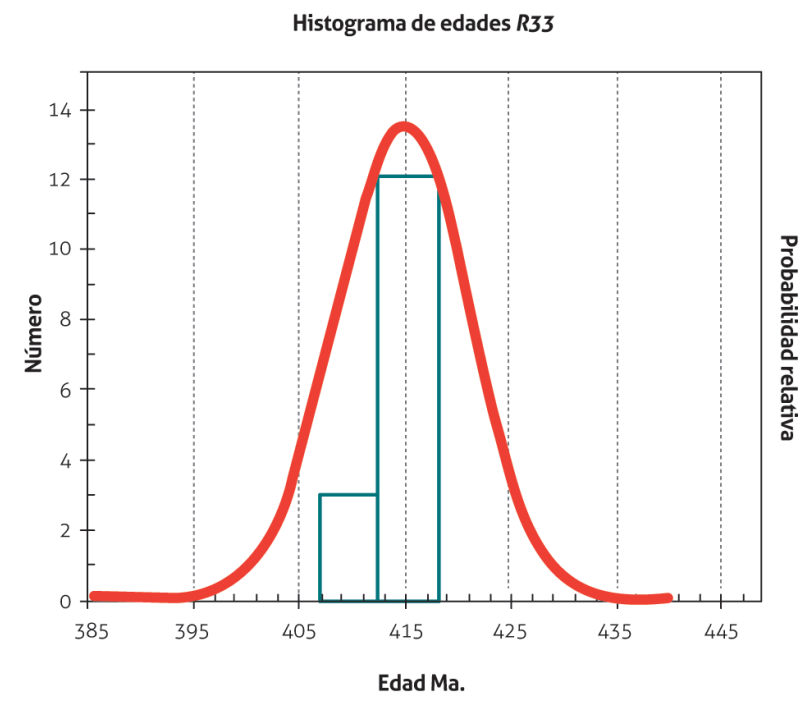

Figura 8. Izquierda, diagrama de concordia Wetherill. Derecha, histograma de edades en material de referencia $R 33$, según el sistema ${ }^{206} \mathrm{~Pb} / 238 \mathrm{U}$ Fuente: autores

\section{e. Fish Canyon Tuff}
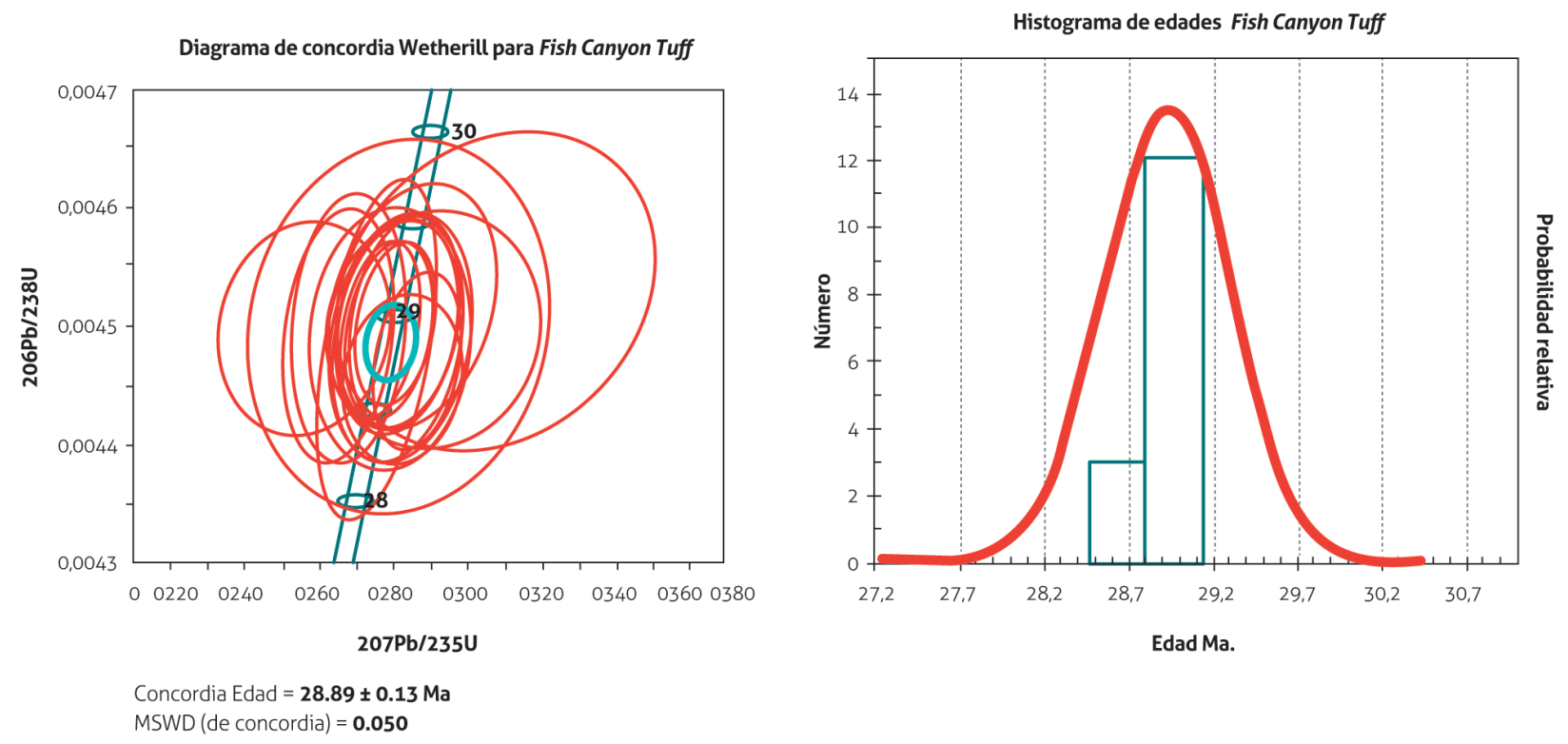

Figura 9. Izquierda, diagrama de concordia Wetherill. Derecha, histograma de edades en material de referencia Fish Canyon Tuff, según el sistema ${ }^{206} \mathrm{~Pb} /{ }^{238} \mathrm{U}$

Fuente: autores 
f. $W F-10$
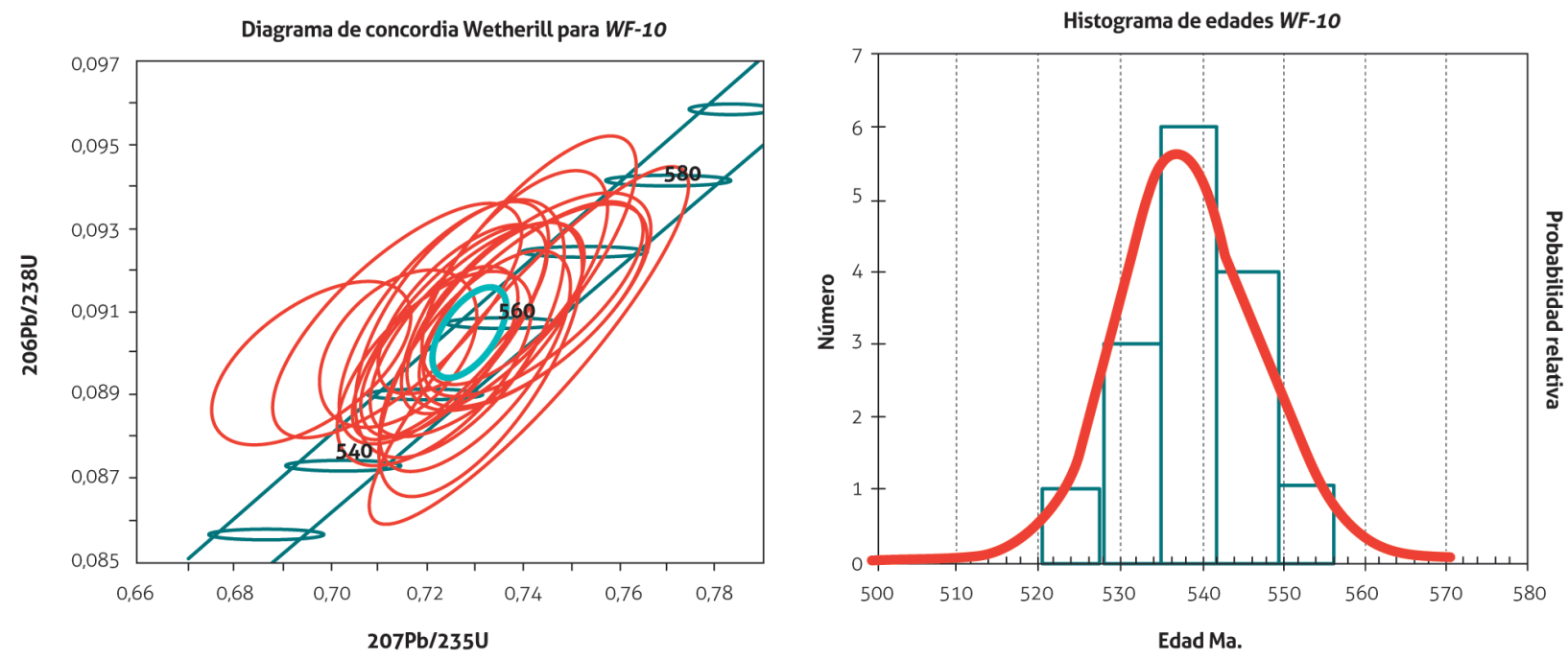

Concordia Edad $=\mathbf{5 5 8 . 1} \pm 3.9 \mathrm{Ma}$

MSWD (de concordia) $=\mathbf{1 . 3}$

Figura 10. Izquierda, diagrama de concordia Wetherill. Derecha, histograma de edades en material de referencia $W F-10$, según el sistema ${ }^{206} \mathrm{~Pb} /{ }^{238} \mathrm{U}$ Fuente: autores

g. WF-6

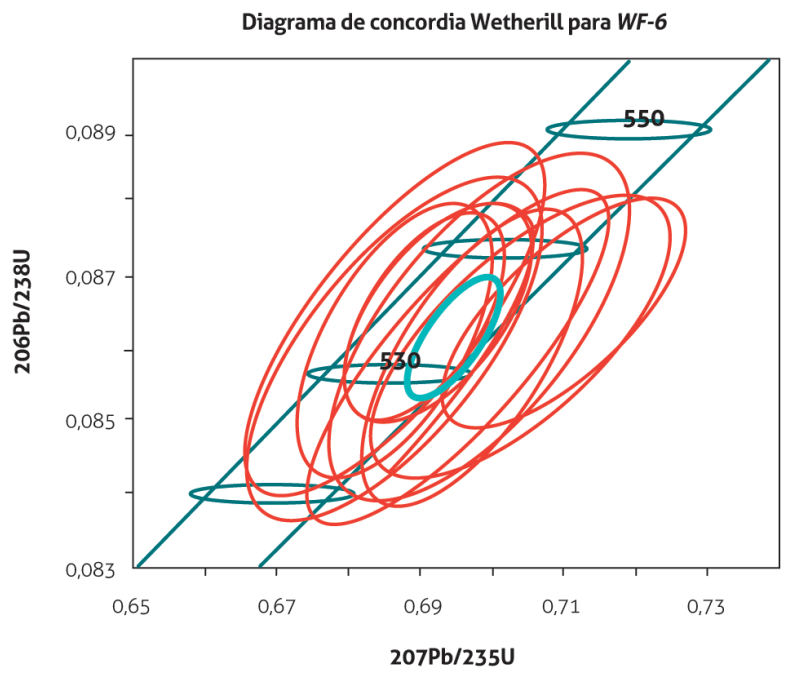

Concordia Edad $=\mathbf{5 3 3} .0 \pm \mathbf{3 . 1} \mathrm{Ma}$ MSWD (de concordia) $=\mathbf{1 . 1 6}$
Histograma de edades WF-6

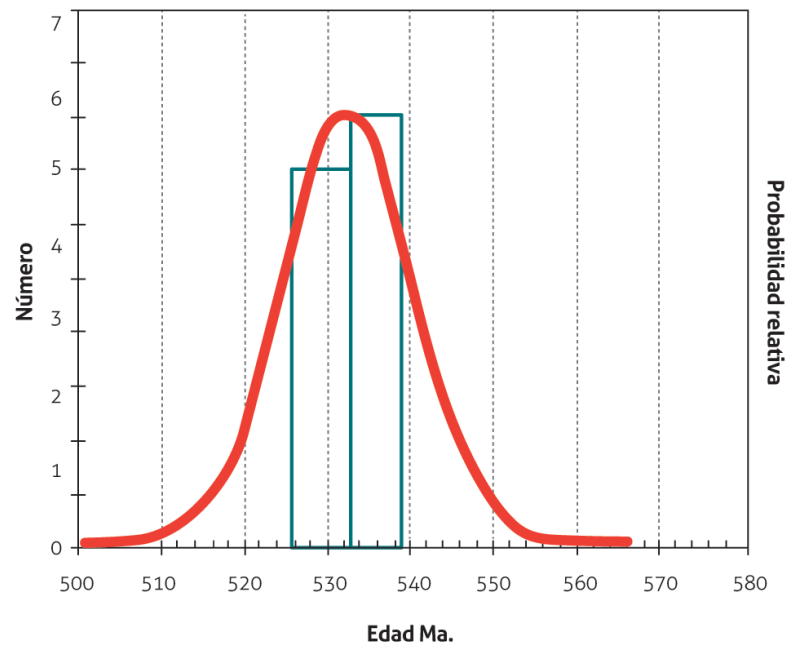

Figura 11. Izquierda, diagrama de concordia Wetherill. Derecha, histograma de edades en material de referencia $W F-6$, según el sistema ${ }^{206} \mathrm{~Pb} /{ }^{238} \mathrm{U}$ Fuente: autores 


\section{h. WF-2}
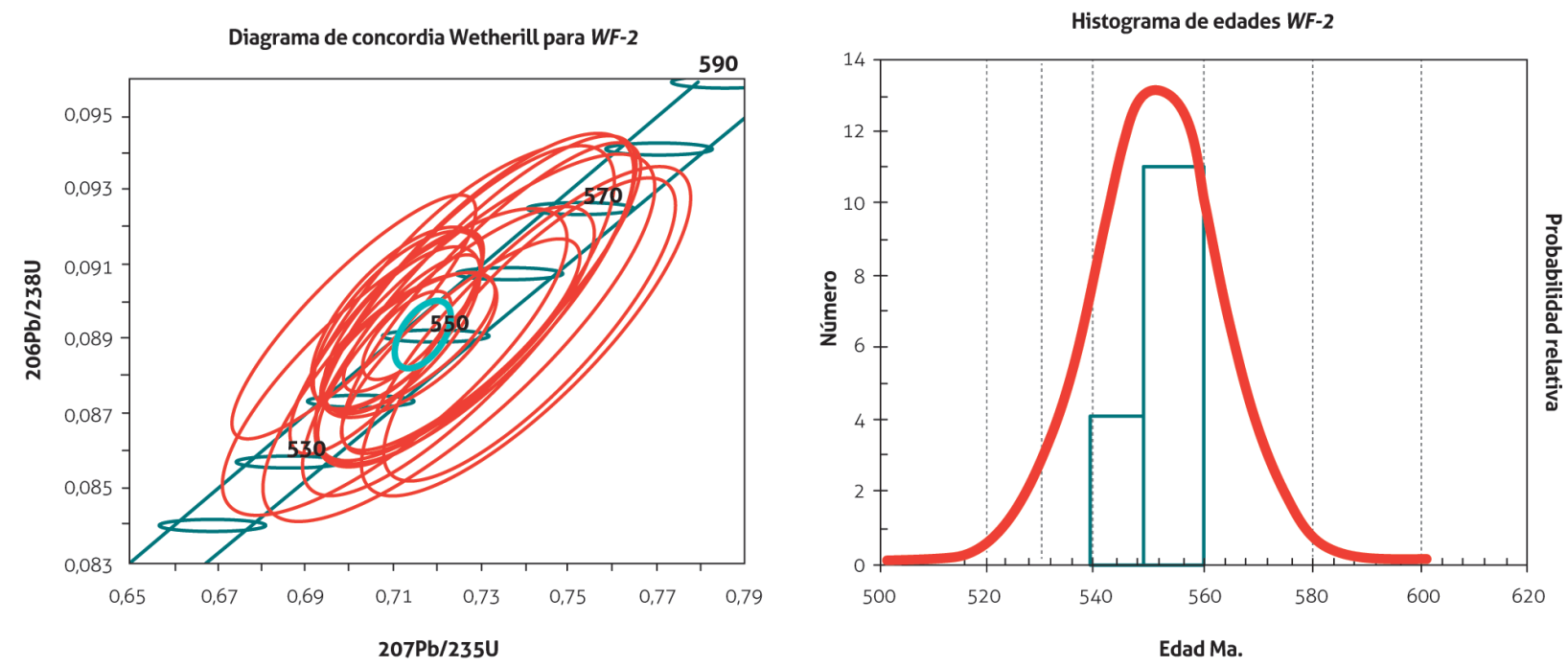

Concordia Edad $=\mathbf{5 5 0 . 6} \pm \mathbf{3 . 2} \mathrm{Ma}$ MSWD (de concordia) $\mathbf{0} \mathbf{0 . 4 1}$

Figura 12. Izquierda, diagrama de concordia Wetherill. Derecha, histograma de edades en material de referencia $W F-2$, según el sistema ${ }^{206} \mathrm{~Pb} /{ }^{238} \mathrm{U}$ Fuente: autores

i. $W F-1$
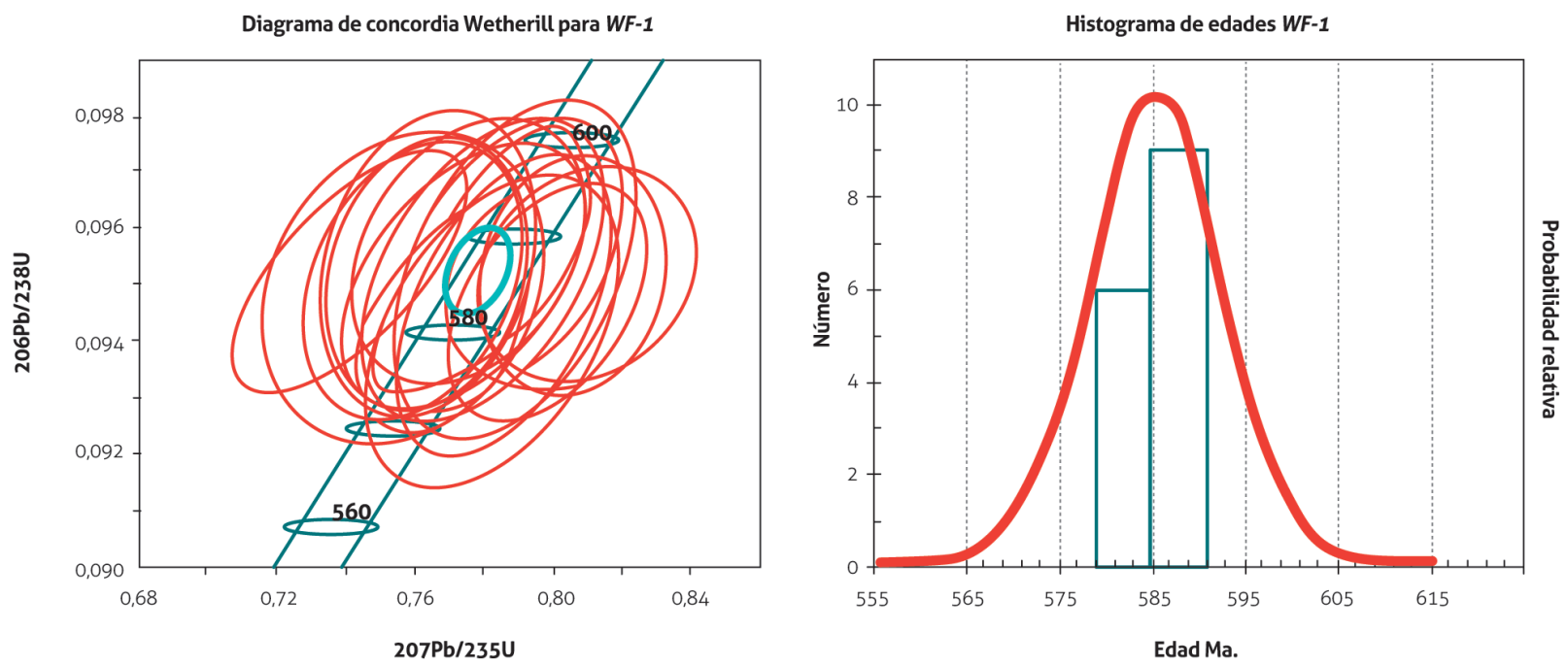

Concordia Edad $=\mathbf{5 8 5 . 9} \pm \mathbf{2 . 9} \mathrm{Ma}$ MSWD (de concordia) $=\mathbf{0 . 2 7}$

Figura 13. Izquierda, Diagrama de concordia Wetherill. Derecha, histograma de edades en material de referencia $W F-1$ según el sistema ${ }^{206} \mathrm{~Pb} /{ }^{238} \mathrm{U}$ Fuente: autores 


\section{j. Resultados finales de la evaluación de estándares}

Tabla 3. Resultados estadísticos correspondientes a los materiales de referencia evaluados. La edad ${ }^{206} \mathrm{~Pb} /{ }^{238} \mathrm{U}$ corresponde al valor promedio de $\mathrm{N}$ cantidad de análisis de cada material de referencia

\begin{tabular}{|c|c|c|c|c|c|}
\hline Muestra & N..$^{\circ}$ de datos & Edad esperada (Ma.) ${ }^{206} \mathrm{~Pb} /{ }^{238} \mathrm{U}$ & Edad obtenida (Ma.) ${ }^{206} \mathrm{~Pb} /{ }^{238} \mathrm{U}$ & * \% exactitud ${ }^{206} \mathrm{~Pb} / 238 \mathrm{U}$ & $\% \% \mathrm{CV}$ (precisión) ${ }^{206} \mathrm{~Pb} / 238 \mathrm{U}$ \\
\hline FC-5 & 24 & $\begin{array}{c}1099 \pm 0,9 \\
\text { (Paces y Miller, 1993) }\end{array}$ & 1109,88 & 0,99 & 0,68 \\
\hline M. Dromedary & 24 & $\begin{array}{c}99,12 \pm 0,14 \\
\text { (Renne et al., 1998) }\end{array}$ & 98,54 & $-0,58$ & 0,51 \\
\hline R33 & 15 & $\begin{array}{c}419,26 \pm 0,39 \\
\text { (Black et al., 2004) }\end{array}$ & 414,79 & $-1,07$ & 0,53 \\
\hline Mud Tank & 15 & $\begin{array}{c}732 \pm 5 \\
\text { (Black y Gulson, 1978) }\end{array}$ & 725,48 & $-0,89$ & 2,04 \\
\hline F. Canyon T. & 15 & $\begin{array}{c}28,402 \pm 0,023 \\
\text { (Lanphere y Baadsgaard, 1999) }\end{array}$ & 28,92 & 1,81 & 0,47 \\
\hline WF-1 & 15 & 590 & 585,46 & $-0,77$ & 0,28 \\
\hline WF-2 & 15 & 560 & 551,72 & $-1,47$ & 0,60 \\
\hline WF-6 & 15 & 530 & 532,44 & 0,46 & 0,36 \\
\hline WF-10 & 15 & 530 & 559,10 & 5,51 & 0,63 \\
\hline
\end{tabular}

* $\quad \%$ error calculado a partir del promedio de edad obtenido del sistema ${ }^{206} \mathrm{~Pb} /{ }^{238} \mathrm{U}$ en cada material de referencia, comparado con la edad esperada según la tabla 1.

* \% CV (coeficiente de variación) calculado a partir de la desviación estándar del conjunto de edades obtenido en cada material de referencia según el sistema ${ }^{206} \mathrm{~Pb} /{ }^{238} \mathrm{U}$. Los valores destacados con color gris están por fuera de los valores esperados.

Fuente: autores

Tabla 4. Resultados de diagramas de concordia Wetherill y MSWD correspondientes a los materiales de referencia evaluados

\begin{tabular}{cccc}
\hline Muestra & Edad de concordia obtenida (Ma.) & t error & MSWD \\
\hline FC 5 & 1110,2 & 4,4 & 0,56 \\
\hline M. Dromedary & 98,53 & 0,33 & 0,0023 \\
\hline R33 & 415,4 & 2,1 & 1,3 \\
\hline Mud Tank & 732,2 & 5,9 & 6,4 \\
\hline F. Canyon T. & 28,89 & 0,23 & 0,050 \\
\hline WF-1 & 585,9 & 2,9 & 0,27 \\
\hline WF-2 & 550,6 & 3,2 & 0,41 \\
\hline WF-6 & 533 & 3,1 & 1,16 \\
\hline WF-10 & 558,1 & 3,9 & 1,3 \\
\hline
\end{tabular}

Fuente: autores

\subsection{Exactitud}

En el momento de evaluar los datos en términos de exactitud en función de los resultados esperados (Euraquem, 2016) de cada uno de los materiales de referencia, en la tabla 3 se observa que la mayoría de los materiales de referencia tiene un porcentaje de error menor del $2 \%$ para la edad ${ }^{206} \mathrm{~Pb} /{ }^{238} \mathrm{U}$, exceptuando el $W-10(5,51 \%)$.
Al promediar los porcentajes de exactitud (como diferencia entre el valor esperado y el valor obtenido en FC 5, Mount Dromedary, R33, Mud Tank, Fish Canyon Tuff, $W F-1, W F-2$ y WF-6, exceptuando los valores de WF-10) se obtiene como valor promedio absoluto una exactitud de aproximadamente $1 \%$, lo cual indica que, en el rango de medición de $100 \mathrm{Ma}$ a $1100 \mathrm{Ma}$, la técnica es exacta para el sistema ${ }^{206} \mathrm{~Pb} /{ }^{238} \mathrm{U}$ debido a que las edades obtenidas son cercanas a las esperadas. De esto se infiere que la metodología utilizada es, en principio, adecuada para la medición de los isótopos de ${ }^{238} \mathrm{U} \mathrm{y}^{206} \mathrm{~Pb}$.

En el caso de $W F-10$, el valor obtenido difiere del valor esperado en un 5,5\% como se puede observar en la figura 10. El histograma revela una clara dispersión de los resultados, con edades desde $520 \mathrm{Ma}$ hasta $560 \mathrm{Ma}$, lo cual probablemente se debe a que son circones que pueden presentar daño por radiación o que no son completamente homogéneos. La falta de exactitud y dispersión de los resultados también puede ser explicada como se describe más adelante, en "Reproducibilidad e histogramas y diagramas de concordia”, esta dispersión de resultados indica que las principales fuentes de error son, probablemente, los parámetros analíticos seleccionados, cuyo control es fundamental para obtener datos menos dispersos y más exactos y precisos. Estos parámetros son frecuencia, energía del láser, tamaño de ablación, orientación 
cristalográfica, plano focal, daño por radiación, reducción de datos y efectos de la matriz. Estos hacen variar el fraccionamiento isotópico, las tasas de ablación y el sesgo de masas, entre otros (Bruguier, 2009; Klotzli et al., 2009; Solari et al., 2010; Marillo-Sialer, Woodhead, Hergt, Greig, Guillong y Gleadow, 2014; Schaltegger, Schmitt y Horstwood, 2015; Solari et al., 2015).

\subsection{Reproducibilidad e histogramas}

Los histogramas correspondientes a los materiales de referencia analizados, en general, tienen un comportamiento similar a una distribución normal con un solo pico cercano a la edad esperada, como se observa en la mayoría de histogramas, con excepción de mud tank y WF-10, cuyos resultados son más dispersos debido a que presentan varias poblaciones de edades, lo cual probablemente se deba a factores analíticos que pueden ocasionar cambios en las relaciones isotópicas que favorecerían el fenómeno de fraccionamiento isotópico elemental. Existen varios parámetros analíticos que pueden ocasionar este fraccionamiento, como la posición de la probeta dentro de la cámara de ablación, la disminución en la fluencia y la frecuencia del láser. Estos factores pueden favorecer el fraccionamiento isotópico y, a la vez, la dispersión de resultados (Bruguier, 2009; Solari, et al., 2010; Solari et al., 2015).

Al revisar la reproducibilidad de la técnica analítica mediante la desviación estándar relativa (\% CV; Euraquem, 2016), reportada en la tabla 3, se observa que, en la mayoría de los materiales de referencia, la dispersión de datos es menor del $2 \%$, como \% CV (desviación estándar relativa). Esto indica que la medición de las concentraciones de cada uno de los isótopos de ${ }^{238} \mathrm{U} y{ }^{206} \mathrm{~Pb}$ durante una rutina analítica para obtener un conjunto de datos es precisa, es decir, que representan una sola población de edades. Sin embargo, en Mud Tank, el \% CV es del $2 \%$, debido a la presencia de varias poblaciones de edades con una mayor dispersión de los datos respecto a la edad esperada. Esta dispersión en las edades obtenidas puede deberse a varios factores, como los siguientes:

- Fluencia y frecuencia: la densidad de energía utilizada $\left(\mathrm{J} / \mathrm{cm}^{2}\right)$ puede ser muy alta, lo cual origina una mayor tasa de ablación y excavación (cráteres más profundos), lo que aumenta el fraccionamiento ele- mental, que es uno de los factores limitantes para la reproducibilidad de la técnica $\mathrm{U}-\mathrm{Pb}$. (Bruguier, 2009; Solari, et al., 2010; Paton et al., 2010; Marillo-Sialer et al., 2014; Schaltegger et al., 2015; Solari et al., 2015).

- Tamaño de ablación: define la resolución espacial de la superficie excavada. Un tamaño de ablación muy pequeño, $<20 \mu \mathrm{m}$, aumenta la probabilidad de fraccionamiento isotópico. En este estudio se utilizó un diámetro de haz de $30 \mu \mathrm{m}$, pero si la densidad de energía es alta, la relación diámetro/profundidad de cráter será mayor de 1 , cuando lo indicado es que sea igual o menor de 1 (Bruguier, 2009; Solari et al., 2010; Paton et al., 2010; Marillo-Sialer et al., 2014; Schaltegger et al., 2015; Solari et al., 2015).

- Otra causa de la dispersión de los resultados es, probablemente, el daño inducido por la radiación en la estructura cristalina debido a la desintegración natural de U y Th (Marillo-Sialer et al., 2014; Solari et al., 2015), que puede ocurrir en los materiales de referencia, como el WF-10, que es un material de referencia comercial de Wild Fish Gems.

\section{a. Diagramas de concordia}

Los diagramas de concordia muestran la diferencia porcentual entre las edades obtenidas por medio de los dos sistemas isotópicos ${ }^{238} \mathrm{U} /{ }^{206} \mathrm{~Pb}$ y ${ }^{207} \mathrm{~Pb} /{ }^{206} \mathrm{~Pb}$ (Wetherill, 1956). Los resultados de los diagramas de concordia (figuras 5 a 13) están resumidos en la tabla 4, y muestran que la edad concordia obtenida en cada uno de los materiales de referencia evaluados es cercana a la esperada, es decir, las edades calculadas mediante los diferentes sistemas isotópicos $\left({ }^{206} \mathrm{~Pb} /{ }^{238} \mathrm{U} y{ }^{207} \mathrm{~Pb} /{ }^{235} \mathrm{U}\right)$ presentan diferencias porcentuales muy bajas o concordantes entre ambos sistemas. Los errores obtenidos al tratar de determinar las edades de concordia son bajos (menores del 1\% respecto a la edad esperada) en todos los materiales de referencia evaluados, lo que indica una buena medición de las relaciones isotópicas y bajo sesgo o errores instrumentales. Esto se logró gracias a la optimización de parámetros como los siguientes:

- Sesgo de masa: este fraccionamiento ocurre porque los electrones producidos durante la ionización en el plasma son eliminados del haz. Los iones restantes se someten a una repulsión positiva, por lo cual los 
isótopos ligeros se dispersan fácilmente, y los isótopos pesados se mueven hacia la trayectoria iónica (Bruguier, 2009). Esto se evita con voltajes adecuados en el SEM, que reducen la diferencia de energía de los iones. Para lograr el voltaje adecuado se utilizó la solución de sintonización, y se monitoreó el

${ }^{113}$ In, al tiempo que se modificaba automáticamente el voltaje del SEM hasta encontrar el voltaje adecuado (punto de inflexión), a partir del cual tiende a ser constante la señal del ${ }^{113} \mathrm{In}$. Tiempo muerto: es el intervalo de tiempo específico durante el cual, después de que el discriminador de pulsos detecta un impacto iónico, el sistema se encuentra inhabilitado para detectar nuevos eventos. No utilizar el tiempo muerto adecuado para el detector del espectrómetro de masas puede ocasionar un sesgo en la medición de los diferentes isótopos y, a la vez, en las relaciones isotópicas, lo cual ocasiona dispersión en los resultados de las edades obtenidas (Nygren, Rameback, Berglund y Baxter, 2006). Para obtener el tiempo muerto adecuado se analizaron varias concentraciones de soluciones de $\mathrm{U}$ desde 0,05 ppb hasta 10,00 ppb, y luego se introdujo el valor obtenido en el ICPMS Element II.

Al analizar la dispersión de datos usando el MSWD obtenido (tabla 4), se evidenció que la mayoría de los materiales de referencia dan resultados adecuados (MSWD $<2$ ). Teniendo en cuenta que el número de análisis realizado para cada material de referencia está entre 15 y 30 datos, el rango aceptable para el MSWD debería encontrarse entre 0,25 y 1,8, como menciona Spencer et al. (2015), con excepción de los resultados obtenidos por mud tank, que indican que el error experimental está siendo subestimado y que, además, los datos no representan una sola población, lo cual se observa en el diagrama de concordia e histograma (figura 7). Por otra parte, los valores de MSWD obtenidos en algunos materiales de referencia (tabla 4, Mount Dromedary y Fish Canyon Tuff) son demasiado bajos (< a 0,01). Según Spencer et al. (2015), esto permite inferir que las incertidumbres analíticas tienen valores muy reducidos, y la edad estaría siendo sobreestimada. Esto obliga a realizar nuevos ensayos, en lo posible probando otras condiciones analíticas con mayor número de datos, para obtener valores adecuados.

\section{b. Comparación de resultados}

Para comparar los resultados obtenidos con los reportados por diferentes autores, como Frei y Gerdes (2009), en la tabla 5 se muestra que los valores obtenidos por el laboratorio son bastante cercanos, lo cual da seguridad respecto a la metodología implementada y brinda alta confiabilidad en los datos generados. La tabla 5 presenta un resumen de los datos correspondientes a los dos materiales de referencia, en los cuales la diferencia significativa se refiere al error de cada valor, que puede ser mejorado aumentando la cantidad de datos. No obstante, el parámetro de evaluación de precisión de los resultados MSWD es muy satisfactorio con respecto a los valores de referencia de Frei y Gerdes (2009). Sin embargo, los errores obtenidos para el promedio de todos los cristales medidos con Mud tank son altos, alrededor del $13 \%$ respecto a la edad de la concordia, por lo cual se deberán evaluar parámetros como fluencia, frecuencia y spot, entre otros, como los descritos, como posibles causantes de la dispersión de los resultados reportados en las secciones 3.1 y 3.2 .

Tabla 5. Comparación de los resultados analíticos con los de otros laboratorios

\begin{tabular}{|c|c|c|c|c|c|c|}
\hline \multirow{2}{*}{ Material de referencia } & \multicolumn{3}{|c|}{ Frei y Gerdes (2009) } & \multicolumn{3}{|c|}{ SGC (este estudio): } \\
\hline & Edad & \pm M.a & MSWD & Edad & \pm M.a & MSWD \\
\hline FC-5 & 1102 & 3 & 10,5 & 1110,2 & 4,4 & 0,56 \\
\hline Mud Tank & 727 & 3 & 0,11 & 732,2 & 5,9 & 6,4 \\
\hline
\end{tabular}

Fuente: autores 


\section{Conclusiones}

Este trabajo representa un avance en la implementación de la técnica de datación U/Pb mediante la LA-ICP-MS en el Laboratorio de Datación U/Pb del Servicio Geológico Colombiano. Se obtuvieron los primeros resultados de materiales de referencia con parámetros establecidos en la literatura geológica internacional, y a partir de los resultados hemos determinado una estrategia para continuar optimizando el método de adquisición y los resultados obtenidos.

Teniendo en cuenta que la precisión de edades $\mathrm{U} / \mathrm{Pb}$ mediante LA-ICPMS es en parte función de a) la estabilidad de las señales de cada masa medida, b) la cantidad de iones captados por el detector, y c) la adecuada estimación de incertidumbre aplicada a las señales medidas (Sláma y Kosler, 2012), es necesario realizar una evaluación de los parámetros analíticos utilizados inicialmente hasta encontrar los valores óptimos y lograr un mejor ajuste de los resultados generados; de tal forma se culminaría la etapa de validación de la técnica de LA-ICPMS en el laboratorio del Servicio Geológico Colombiano para datación $\mathrm{U} / \mathrm{Pb}$.

Es recomendable realizar la optimización de parámetros como la frecuencia, fluencia y spot, con el fin de encontrar la mejor relación de los tres para evitar el fraccionamiento isotópico que, en principio, genera resultados dispersos y con incertidumbres analíticas altas. De igual forma, se deberán controlar otros posibles causantes de la dispersión de los resultados, como enfoque y plano focal de las muestras y fraccionamiento isotópico entre distintas posiciones de la probeta en la cámara de ablación, entre otras.

Los resultados analíticos evaluados mediante el parámetro MSWD muestran que el laboratorio genera datos de baja dispersión, lo que garantiza una precisión y exactitud adecuada, según los criterios internacionales, al comparar los resultados con los reportados por otros autores, como Frei y Gerdes (2009).

Estos primeros resultados, obtenidos mediante la técnica LA-ICP-MS en el Laboratorio del Servicio Geológico Colombiano, permiten establecer que el laboratorio está en capacidad de obtener resultados precisos y exactos, aunque como proceso de mejora se requiere optimizar algunos parámetros de análisis, como los mencionados anteriormente. El desarrollo e implementación de esta técnica analítica significa un gran aporte a los diferentes proyectos del Servicio Geológico Colombiano relacionados con geocronología que involucran análisis de proveniencia, datación de diferentes tipos de rocas para la definición de unidades litológicas y diferenciación de eventos geológicos.

\section{Agradecimientos}

El proceso de implementación de la técnica analítica contó con la participación inicial de los expertos internacionales de Thermo Fischer Scientific, Dr. Tony Cade y Dr. Henry Robert. Además, se contó con el Dr. Jeremy Hourigan, de la Universidad de Stanford, actualmente profesor asistente de la Universidad de Santa Cruz, experto en la técnica de datación $\mathrm{U}-\mathrm{Pb}$ aplicada en geociencias asociadas a los estudios sobre amenazas, geotermia, recursos del subsuelo, volcanes, cartografía y geología básica, que requieren información geocronológica para adelantar los estudios geológicos y de exploración que actualmente se realizan en el país.

\section{RefERENCIAS BIBLIOGRÁFICAS}

Black, L. y Gulson, B. (1978). The age of the Mud Tank Carbonatite, Strangways Range, Northern Territor. BMR Journal of Australian Geology \& Geophysics, 3(3), 227-232.

Black, L., Kamo, S. L. y Allen, C. M. (2004). Improved $\mathrm{Pb}-206 / \mathrm{U}-218$ microprobe geochronology by the monitoring of a trace-element-related matrix effect; SHRIMP, ID-TIMS, ELA-ICP-MS and oxygen isotope documentation for a series of zircon standards. Chemical Geology, 205(6), 115-140. Doi: 10.1016/j.chemgeo.2004.01.003.

Bouman, C., Schwieters, J., Cocherie, A., Robert, M. y Wieser, M. (2014). In situ U-Pb zircon dating using laser ablation - multi ion counting - ICP - MS (LA-MICICP-MS). Aplication note. Thermo Fisher Scientific. Disponible en https://tools.thermofisher.com/content/sfs/brochures/AN-30021-ICP-MS-U-Pb-Zircon-Dating-AN30021-EN.pdf.

Bruguier, O. (2009). Géochronologie U-Pb par ablation laser et ICP-MS (LA-ICP-MS): Principes, Complexités et Perspectives (tesis). Universidad de Montpellier II, Montpellier, Francia. 
Campbell, I. H., Reiners, P. W. y Allen, C. M. (2005). He$\mathrm{Pb}$ double dating of detrital zircons from the Ganges and Indus Rivers: Implication for quantifying sediment recycling and prevenance studies. Earth and Planetary Sciencie Letters, 237(3-4), 402-432. Doi: 10.1016/j.epsl.2005.06.043.

Chang, Z., Vervoort, J. D., McClelland, W. C. y Knaack, C. (2006). U-Pb dating of zircon by LA-ICP-MS. Geochemestry Geophysics Geosystems, 7(5), 1-14. Doi: 10.1029/2005GC001100.

Cherniak, D. J. y Watson, E. B. (2001). Pb diffusion in zircon. Chemical Geology, 172, 5-24.

EURAQUEM/CITAC. (2016). Guide to Quality in Analytical Chemistry an Aid to Accreditation. Disponible en www.eurachem.org.

Frei,D.yGerdes, A. (2009). Preciseandaccuratein situU-Pb dating of zircon with high sample throughput by automated LA-SF-ICP-MS. Chemical Geology, 261(3-4), 261-270. Doi: 10.1016/j.chemgeo.2008.07.025.

Gehrels, G., Valencia, V. A. y Ruiz, J. (2008). Enhanced precision, accuracy, efficiency, and spatial resolution of $\mathrm{U}-\mathrm{Pb}$ ages by laser ablation-multicollector-inductively coupled plasma-mass spectrometry. Geochemistry, Geophysics, and Geosystems, 9(3). Doi: 10.1029/2007GC001805.

Hellstrom, J. E. (2008). Iolite: Software for spatially resolved LA- (quad and MC) ICP-MS analysis. En P. Sylvester (Ed.), Laser Ablation ICP-MS in the Earth Sciences: Current Practices and Outstanding Issues. Vancouver: Mineralogical Association of Canada.

Horstwood, M. (2008). Data reduction strategies, uncertainty assessment and resolution of LA-(MC-) ICPMS isotope data. En P. Sylvester (Ed.), Laser ablationICP-MS in the Earth Sciences: Current practices and outstanding issues. Vancouver: Mineralogical Association of Canada.

Horstwood, M., Kosler, J., Gehrels, G., Jackson, S. y McLean, N. M. (2016). Community-derived standards for LA-ICP-MS U-(Th-)Pb geochronology - uncertainty propagation, age interpretation and data reporting. Geostandards and Geoanalytical Research, 40(3), 311-332. Doi: 10.1111/j.1751-908X.2016.00379.x.

Jackson, S. E., Pearson, N. J., Griffin, W. L. y Belousova, E. A. (2004). The application of laser ablation-inductively coupled plasma-mass spectrometry to in situ U-Pb zircon geochronology. Chemical Geology, 211(1-2), 47-69. Doi: 10.1016/j.chemgeo.2004.06.017.

Jaffey, A. H. (1971). Precision measurement of half - lives and specific activities of $235 \mathrm{U}$ and 238U. Physical Review C, 4(5), 1889-1906. Doi: 10.1103/PhysRevC.4.1889.

Klotzli, U., Klotzli, E., Gunes, Z. y Kosler, J. (2009). Accuracy of laser ablation U-Pb zircon dating: Results from a test using five different reference zircons. Geostandards and Geoanalytical Research, 33(1), 5-15. Doi: 10.1111/j.1751-908X.2009.00921.x.

Kosler, J. (2007). Laser Ablation IPC-MS - a new dating tool in Earth Science. Proceedings of the Geologist Asoociation, 118(1), 19-24. Doi: 10.1016/S00167878(07)80043-5.

Kosler, J. y Sylvester, P. J. (2003). Present trends and the future of zircon in geochronology: Laser ablation ICPMS. Reviews in Mineralogy and Geochemistry, 53(1), 243-275. Doi: 10.2113/0530243.

Lanphere, M. y Baadsgaard, H. (1999). The Fish Canyon Tuff: A standard for geochronology. Bulletin of Liaison and Informations, Phanerozoic Time Scale, 15(2), 20-21.

Lee, M. S. (2012). Mass Spectrometry Handbook (Vol. 1). New Jersey: Wiley.

Ludwig, K. R. (2012). User's manual for Isoplot 3.75: A geochronological toolkit for Microsoft Excel. Special Publication, Issue 5. Berkeley: Berkeley Geochronology Center.

Marillo-Sialer, E., Woodhead, J., Hergt, J., Greig, A., Guillong, M. y Gleadow, A. (2014). The zircon 'matrix effect': Evidence for an ablation rate control on the accuracy of U-Pb age determinations by LA-ICP-MS. Journal of Analytical Atomic Spectrometry, 29(6), 9431140. Doi: 10.1039/C4JA00008K.

Mattinson, J. M. (2005). Zircon U-Pb chemical abrasion (“CA-TIMS”) method: Combined annealing and multi-step partial dissolution analysis for improved precision and accuracy of zircon ages. Chemical Geology, 220(1-2), 47-66. Doi: 10.1016/j.chemgeo.2005.03.011. Moser, D. y Scott, D. (2001). Towards a more accurate U$\mathrm{Pb}$ geochronology. Chemical Geology, 172(1-2), 1-3. Doi: 10.1016/S0009-2541(00)00232-1.

Nygren, U., Rameback, H., Berglund, M. y Baxter, D. C. (2006). The importance of a correct dead time setting 
in isotope ratio mass spectrometry: Implementation of an electronically determined dead time to reduce measurement uncertainty. International Journal of Mass Spectrometry, 257(1-3), 12-15. Doi: 10.1016/j. ijms.2006.05.011.

Paces, J. B. y Miller Jr., J. D. (1993). Precise U-Pb ages of Duluth Complex and related mafic intrusions, northeastern Minnesota: geochronological insights into physical, petrogenetic, paleomagnetic and tectonomagmatic processes associated with the $1.1 \mathrm{Ga}$ Midcontinent Rift System. Journal of Geophysical Research, 98(B8), 13997-14013. Doi: 10.1029/93JB01159.

Paton, C., Woodhead, J. D. Hellstrom, J. C. Hergt, J. M., Greig, A. y Maas, R. (2010). Improved laser ablation $\mathrm{U}-\mathrm{Pb}$ zircon geochronology through robust downhole fractionation correction. Gechemistry Geophysics Geosystems, 11(3), 1-36. Doi: 10.1029/2009GC002618.

Pearce, N. J. G., Perkins, W. T., Westgate, J. A., Gorton, M. P., Jackson, S. E. y Neal, C. R. (1997). A compilation of new and published major and trace element data for NIST SRM 610 and NIST SRM 612 glass reference materials. Geostandards and Geonalytical Research, 21(1), 115-144. Doi: 10.1111/j.1751-908X.1997.tb00538.x.

Putirka, K. (2015). Data, ideas, and the nature of scientific progress. American Mineralogist, 100(8-9), 16571658. Doi: 10.2138/am-2015-Ed1008-94.

Renne, P. R., Swisher, C. C., Deino, A. L., Karner, D. V., Owens, T. L. y DePaolo, D. J. (1998). Intercalibration of standards, absolute ages and uncertainties in 40Ar/39Ar dating. Chemical Geology, 145(1-2), 117152. Doi: 10.1016/S0009-2541(97)00159-9.

Schaltegger, U., Schmitt, A. K. y Horstwood, M. (2015). $\mathrm{U}-\mathrm{Th}-\mathrm{Pb}$ zircon geochronology by ID-TIMS, SIMS, and laser ablation ICP-MS: Recipes, interpretations, and opportunities. Chemical Geology, 402, 89-110. Doi: 10.1016/j.chemgeo.2015.02.028.

Schoene, B. (2014). U-Th-Pb Geochronology. En K. K. Turekian y H. D. Holland (Eds.), Treatise on Geochemistry. Vol. 4. Oxford: Elsevier.
Sláma, J. y Kosler, J. (2012). Effects of sampling and mineral separation on accuracy of detrital zircon studies. Geochemistry, Geophysics, Geosystems, 13(5), Q05007. Doi: 10.1029/2012GC004106.

Sláma, J. Kosler, J., Condon, D. J., Crowley, J. L., Gerdes, A. y Hanchar, J. M. (2008.). Plešovice zircon - A new natural reference material for $\mathrm{U}-\mathrm{Pb}$ and $\mathrm{Hf}$ isotopic microanalysis. Chemical Geology, 249(1-2), 1-35. Doi: 10.1016/j.chemgeo.2007.11.005.

Solari, L. A., Gómez-Tuena, A., Bernal, J. P., Pérez-Arvizu, O. y Tanner, M. (2010). U-Pb Zircon Geochronology with an Integrated LA-ICP-MS Microanalytical Workstation: Achievements in Precision and Accuracy. Geostandards and Geoanalytical Research, 34(1), 5-18. Doi: 10.1111/j.1751-908X.2009.00027.x.

Solari, L. A., Ortega-Obregón, C. y Bernal, J. P. (2015). $\mathrm{U}-\mathrm{Pb}$ zircon geochronology by LAICPMS combined with thermal annealing: Achievements in precision and accuracy on dating standard and unknown samples. Chemical Geology, 414, 109-123. Doi: 10.1016/j. chemgeo.2015.09.008.

Spencer, C. J., Kirkland, C. L. y Taylor, R. J. (2015). Strategies towards statiscally robust interpretations of in situ U-Pb zircon geochronology. Geoscience Frontiers, 7(4), 581-589. Doi: 10.1016/j.gsf.2015.11.006.

Sylvester, P. J. (2008). Laser ablation-ICP-MS in the Earth Sciences: Current practices and outstanding issues. Vancouver: Mineralogical Association of Canada. Geostandards and Geoanalytical Research, 29(1), 4152. Doi: 10.1111/j.1751-908X.2005.tb00654.x.

Wendt, I. y Carl, C. (1992). The statistical distribution of the mean squared weighted deviation - Reply. Chemical Geology, 94(3), 242-243. Doi: 10.1016/S00092541(10)80009-9.

Wetherill, W. G. (1956). Discordant Uranium - Lead Ages, I. Earth \& Space Science News, 37(3), 320-326. Doi: 10.1029/TR037i003p00320. 